\title{
Antibiotika versagen in der ambulanten Behandlung
}

Die Antibiotikaresistenz von Keimen hat in den letzten

Jahrzehnten dramatisch zugenommen. Ein wichtiger

Risikofaktor für eine Infektion mit resistenten Keimen stellt

die ambulante Behandlung dar. Daher haben C. J. Currie et al. das Therapieversagen bei 4 üblichen Infektionen im

ambulanten Bereich untersucht.

BMJ 2014; 349: g5493

Aus 58 Millionen Antibiotikaverschreibungen, die in der Datenbank Clinical Practice Research Datalink zwischen 1991 und 2012 verzeichnet wurden, wählten die Autoren fast 11 Mio. Monotherapien für eine der folgenden Indikationen aus: $38,6 \%$ der Therapien für Infektionen des oberen und $28,7 \%$ für solche des unteren Respirationstraktes, 23,4\% für Haut- und Weichteilinfektionen und 9,2\% für akute Otitis media. Insgesamt lag im Jahr 2012 die Versagensquote bei $15,4 \%$.

Für den Vergleich zwischen den Jahren wurden die Versagensraten relativ zum Wert des Jahres 1991 (entspricht 100) standardisiert. Daraus ergibt sich ein Anstieg des Gesamtversagens um 12\% im Vergleich zu 1991 (112, 95\% Konfidenzintervall [KI] 112-113). Die höchste Versagensrate wurde bei Infektionen des unteren Respirationstraktes beobachtet (135, 95\%-KI 134-136). Während die Versagensrate der am häufigsten verschriebenen Antibiotika (Amoxicillin, Penicillin V und Flucloxacillin) unter $20 \%$ lag, gab es bedeutsame Anstiege für Trimethoprim bei der Behandlung von Infektionen der oberen Atemwege (von 29,2\% zwischen 1991-1995 auf 70,1\% zwischen 20082012) und für Ciprofloxacin (von 22,3 auf $30,8 \%$ ) sowie für Cefalexin (von 22,0 auf $30,8 \%$ ) in der Behandlung von Infekten des unteren Respirationstraktes. Die Versagensraten für Breitbandpenicilline, Makrolide und Flucloxacillin blieben weitgehend stabil.

Betrachtet man alle Antibiotikaverordnungen in dieser Zeit, handelte es sich in 98\% der Fälle um eine Monotherapie. Aufgrund der Datenerhebungen im ambulanten Bereich sind keine Angaben zu In-vit-
ro-Keimresistenzen als Ursache für einen Antibiosewechsel verfügbar. Patienten, die wegen Infekten der unteren Atemwege behandelt wurden, waren mit einem mittleren Alter von 48,9 Jahren in der frühen und 52,1 Jahren in der späteren Erhebungszeit am ältesten. Sie stellten zudem das kränkste Kollektiv dar, mit dem höchsten Durchschnitts-BMI (25,8 auf 28,3 ansteigend), dem höchsten Blutdruck (138,4/80,6 bzw. $131,7 / 76,6 \mathrm{mmHg})$, waren am häufigsten Raucher (26,7\% abnehmend auf später $23,3 \%$ ) und erhielten eine Steroid- und Bronchodilatatorentherapie (verdoppelt während der Beobachtungszeit).

Das am häufigsten verschriebene Medikament war Amoxicillin (42,4\% der selektierten Infektionen), gefolgt von Penicillin V (11,8\%; hierbei 95\% für Infektionen der oberen Atemwege) und Flucloxacillin (11,1\%; hiervon $97 \%$ für Haut- und Weichteilinfektionen). Die meisten wahrscheinlichen Versagensfälle (94,4\%) wurden über das Kriterium des Antibiotikawechsels innerhalb von 30 Tagen identifiziert.

\section{Fazit}

Zusammenfassend versagte mehr als eines von 10 Antibiotika, die für eine der 4 Indikationen zwischen 1991 und 2012 verschrieben wurden. Die Rate des Therapieversagens stieg um $12 \%$ in dieser Zeit. Die Ergebnisse implizieren, dass die niedergelassenen Kollegen eine zentrale Rolle bei der Eingrenzung der Versagensraten haben könnten, indem sie sorgfältig entscheiden, welche Antibiotikatherapie gerechtfertigt ist, so die Autoren.

Dr. Susanne Bossenmayer, Stuttgart 